\title{
ON THE REPRESENTATION SYSTEMS WITH RESPECT TO SUMMATION METHODS
}

\author{
K. S. KAZARIAN \\ Departamento de Matemáticas, $C$-XV Universidad Autonoma de Madrid \\ Madrid, 28049, Spain \\ E-mail: kazaros.kazarian@uam.es
}

\begin{abstract}
Properties of representation systems with respect to summation methods are studied. For a given representation system with respect to a given summation method we study, in particular, the question of the stability of that property after deleting finitely many elements. As a consequence we obtain the existence of null series for the systems with respect to a given method of summation.
\end{abstract}

1. Introduction. The motivation of the present study comes from the following two theorems (see [6]-[8]) proved recently.

THEOREM 1. There exists a complete ONS $\Omega=\left\{\Omega_{n}\right\}_{n=1}^{\infty},\left\|\Omega_{n}\right\|_{L_{[0,1]}^{\infty}} \leq M$ for all $n \in \mathbf{N}$, such that $\sum_{n=1}^{\infty} a_{n} \Omega_{n}$ converges a.e. on $[0,1]$ if $\left\{a_{n}\right\}_{n=1}^{\infty} \in l^{2}$ and $\sum_{n=1}^{\infty} a_{n} \Omega_{n}$ diverges a.e. for any $\left\{a_{n}\right\}_{n=1}^{\infty} \notin l^{2}$.

THEOREM 2. Let $\left\{f_{n}\right\}_{n=1}^{\infty}$ be a complete ONS of functions defined on [0,1]. Then there exists an increasing subsequence $\left\{n_{k}\right\}_{k=1}^{\infty}$ of natural numbers such that for any measurable and a.e. finite function defined on $[0,1]$ and some sequence of coefficients $\left\{b_{n}\right\}_{n=1}^{\infty}$

$$
\sum_{n=1}^{n_{k}} b_{n} f_{n}(x) \rightarrow f(x) \quad \text { a.e. when } k \rightarrow \infty .
$$

It thus becomes of interest to study the properties of a system of functions $F=$ $\left\{f_{k}\right\}_{k=1}^{\infty}$ such that any measurable and a.e finite function can be represented by a series $\sum_{k=1}^{\infty} a_{k} f_{k}$ which is a.e. $T$-summable, where $T$ is a fixed summation method, to the function $f$. Traditionally one of the first questions that have been studied is the existence of null series. Having Theorem 2 one would like to clarify if for any complete ONS $\left\{f_{n}\right\}_{n=1}^{\infty}$ of functions defined on $[0,1]$ there exists an increasing subsequence $\left\{n_{k}\right\}_{k=1}^{\infty}$ of natural

2000 Mathematics Subject Classification: Primary 42C15.

The author was partially supported by grants MTM2004-00678 and HH 2004/0002.

The paper is in final form and no version of it will be published elsewhere. 
numbers and a non-trivial series $\sum_{n=1}^{\infty} a_{n} f_{n}(x)$ such that

$$
\sum_{n=1}^{n_{k}} a_{n} f_{n}(x) \rightarrow 0 \quad \text { a.e. when } k \rightarrow \infty \text {. }
$$

We study this question in a general and more abstract context. The formulation of our main result needs various definitions which are given in the next section. Here we give some corollaries of the main result. In particular, the answer to the question formulated above.

Proposition 3. Let $\left\{f_{n}\right\}_{n=1}^{\infty}$ be a complete ONS of functions defined on [0,1] and $\left\{n_{k}\right\}_{k=1}^{\infty}$ be an increasing subsequence of natural numbers such that for any measurable and a.e. finite function defined on $[0,1]$ and some sequence of coefficients $\left\{b_{n}\right\}_{n=1}^{\infty}$ we have (1). Then there exists a non-trivial series $\sum_{n=1}^{\infty} a_{n} f_{n}(x)$ such that (2) holds.

PROPOSITION 4. Let $T$ be a regular triangular matrix (see section 2) and let $\left\{\varphi_{n}\right\}_{n=1}^{\infty}$ be a system of functions defined on $[0,1]$ such that for any measurable and a.e. finite function defined on $[0,1]$ and some sequence of coefficients $\left\{b_{n}\right\}_{n=1}^{\infty}$ the series $\sum_{n=1}^{\infty} b_{n} f_{n}(x)$ is Tsummable a.e. to the function $f$. Then for any natural number $N$ the system $\left\{\varphi_{n}\right\}_{n=N+1}^{\infty}$ has the same property.

Proposition 4 is an extension of a result by A. A. Talalian [11] for summation methods. It should be mentioned that representation of functions by trigonometric series that are summable in Abel-Poisson and some other methods were considered by N. N. Lusin [9]. He also has indicated the interest of studying such questions for other orthogonal systems. It seems that after Menshov's [10] proof that the trigonometric system is a system of convergence in the sense of a.e. convergence mainly the ordinary convergence was studied in the theory of representation of function by series. The following remark at the end of B. S. Kashin's article [4] should be mentioned as an exception: “... it is possible to construct a system $\left\{\psi_{n}(x)\right\}$ such that the series $\sum_{n=1}^{\infty} c_{n} \psi_{n}(x)$ is not summable on sets of positive measure if its coefficients satisfy condition $\sum_{n=1}^{\infty} c_{n}^{2}=\infty$." We do not know if there was any publication with specification of those summation methods.

2. Definitions and preliminary results. A quasi-norm (or Fréchet norm) on a linear space $X$ is a real-valued function $\|\cdot\|$ on $X$ such that

$$
\begin{aligned}
\|\mathbf{x}\| & \geq 0 \text { and }\|\mathbf{x}\|=0 \text { if and only if } \mathbf{x}=0 \\
\|\mathbf{x}+\mathbf{y}\| & \leq\|\mathbf{x}\|+\|\mathbf{y}\|, \\
\|-\mathbf{x}\| & =\|\mathbf{x}\|, \lim _{\alpha_{n} \rightarrow 0}\left\|\alpha_{n} \mathbf{x}\right\|=0, \text { and } \lim _{\left\|\mathbf{x}_{n}\right\| \rightarrow 0}\left\|\alpha \mathbf{x}_{n}\right\|=0
\end{aligned}
$$

where $\alpha$ denotes a scalar. A complete quasi-normed linear space is called an $F$-space (or Fréchet space) [12], pp. 30-31, 52. The real and natural numbers will be denoted by $\mathbf{R}$ and $\mathbf{N}$ respectively. Let $\chi_{E}$ denote the characteristic function of a set $E \subseteq[0,1]$.

Let $M$ be the linear space of all Lebesgue measurable real-valued finite almost everywhere functions on the interval $[0,1]$ and let $M_{0}$ denote the subspace of functions almost everywhere equal to zero. We consider $F$-spaces with elements in the factor space $M / M_{0}$. An $F$-space $\mathbf{F}$ is said to be a Fréchet function space (see also [5]) if 
(i) $\mathbf{F} \subseteq M / M_{0}$ and $\mathbf{x}$ is the trivial element of $\mathbf{F} \Leftrightarrow \mathbf{x} \equiv M_{0}$;

(ii) $|g| \leq|f|$ a.e. and $f+M_{0} \in \mathbf{F} \Rightarrow g+M_{0} \in \mathbf{F}$ and $\left\|g+M_{0}\right\| \leq\left\|f+M_{0}\right\|$.

We would like to indicate the parallelism of the introduced term with the definition of Banach function spaces (see [2]).

Further in the paper we will write $f \in \mathbf{F}$ instead of $f+M_{0} \in \mathbf{F}$. By $L_{[0,1]}^{0}$ we will denote linear space $M / M_{0}$ with the quasi-norm

$$
\|f\|_{L_{[0,1]}^{0}}=\int_{0}^{1} \frac{|f(x)|}{1+|f(x)|} d x .
$$

Let $T=\left(t_{i j}\right)_{i, j \in \mathbf{N}}$ be a regular triangular matrix (see [13], [3]): $t_{i j}=0$ if $j>i$ and

$$
\begin{array}{ll}
\sum_{j=1}^{\infty}\left|t_{i j}\right| \leq C<\infty & \forall i \in \mathbf{N} ; \\
\lim _{i \rightarrow \infty} t_{i j}=0 & \forall j \in \mathbf{N} ; \\
\lim _{i \rightarrow \infty} \sum_{j=1}^{\infty} t_{i j}=1 . &
\end{array}
$$

For a given system of functions $\Phi=\left\{\phi_{k}\right\}_{k=1}^{\infty}$ defined on $[0,1]$ and a given sequence of coefficients $\mathbf{c}=\left\{c_{k}\right\}_{k=1}^{\infty}$ we denote

$$
S_{n}(\mathbf{c}, \Phi)(x)=\sum_{k=1}^{n} c_{k} \phi_{k}(x)
$$

and

$$
\sigma_{n}(\mathbf{c}, \Phi, T)(x)=\sum_{j=1}^{n} t_{n j} S_{j}(\mathbf{c}, \Phi)(x) .
$$

Definition 5. Let $\mathbf{F}$ be a Fréchet function space. A system of functions $\Phi=\left\{\phi_{k}\right\}_{k=1}^{\infty}$ defined on $[0,1]$ is called an $(M, \mathbf{F})$-representation system of the space $L_{[0,1]}^{0}$ if, for every $f \in L_{[0,1]}^{0}$, there exist a sequence of coefficients $\mathbf{c}$ and a bounded function $m_{f}, 0<$ $m_{f}(x) \leq 1$ for $x \in[0,1]$ such that

$$
S_{n}\left(\mathbf{c}, m_{f} \Phi\right)(\cdot) \stackrel{\mathbf{F}}{\rightarrow} m_{f}(\cdot) f(\cdot), \quad \text { when } \quad n \rightarrow \infty,
$$

where $m_{f} \Phi:=\left\{m_{f} \phi_{k}\right\}_{k=1}^{\infty}$.

Definition 6. Let $\mathbf{F}$ be a Fréchet function space. A system of functions $\Phi=\left\{\phi_{k}\right\}_{k=1}^{\infty}$ defined on $[0,1]$ is called a $T$ - $(M, \mathbf{F})$-representation system of the space $L_{[0,1]}^{0}$ if, for every $f \in L_{[0,1]}^{0}$, there exist a sequence of coefficients $\mathbf{c}$ and a bounded function $m_{f}, 0<$ $m_{f}(x) \leq 1$ for $x \in[0,1]$ such that

$$
\sigma_{n}\left(\mathbf{c}, m_{f} \Phi, T\right)(\cdot) \stackrel{\mathbf{F}}{\rightarrow} m_{f}(\cdot) f(\cdot), \quad \text { when } n \rightarrow \infty
$$

and thus

$$
\sigma_{\mathbf{F}}^{*}\left(\mathbf{c}, m_{f} \Phi, T\right):=\sup _{n}\left\|\sigma_{n}\left(\mathbf{c}, m_{f} \Phi, T\right)(\cdot)\right\|_{\mathbf{F}}<+\infty .
$$

The following result of Banach ([1], p. 25) will be used in the proof of Theorem 9. 
LEMma 7 (Banach). Let $U$ be a continuous linear operator defined on an $F$-space $\mathbf{E}$ whose image is another $F$-space $\mathbf{E}_{1}$. Then for every $\varepsilon>0$ there is an $\eta>0$ such that the image of the open sphere $\left\{x \in E:\|x\|_{\mathbf{E}}<\varepsilon\right\}$ under $U$ contains the open sphere $\left\{x \in E_{1}:\|x\|_{\mathbf{E}_{1}}<\eta\right\}$.

3. On the stability of representation systems. Let $\Phi=\left\{\phi_{k}\right\}_{k=1}^{\infty}$ be a $T$ - $(M, \mathbf{F})$ representation system of the space $L_{[0,1]}^{0}$. Then for any $f \in L_{[0,1]}^{0}$ we denote by $C_{f}(\Phi)$ the class of all coefficients $\mathbf{c}=\left\{c_{k}\right\}_{k=1}^{\infty}$ such that for any $\mathbf{c}$ there exists a bounded function $m_{f}, 0<m_{f}(x) \leq 1$ for $x \in[0,1]$ for which (3) is satisfied. For any $\mathbf{c} \in C_{f}(\Phi)$ denote by $\mathcal{M}(\mathbf{c}, f)$ the class of all functions $m_{f} \in L_{[0,1]}^{\infty}, 0 \leq m_{f}(x) \leq 1$ for which (3) is satisfied. For any $0<\alpha \leq 1$ let us introduce also $\mathcal{M}_{\alpha}$ : the class of all functions $m \in L_{[0,1]}^{\infty}, 0 \leq m(x) \leq 1$ such that

$$
|\{x \in[0,1]: m(x)=1\}| \geq 1-\alpha .
$$

For a given $f \in L_{[0,1]}^{0}$ and any $\mathbf{c} \in C_{f}(\Phi)$ let

$$
\|\mathbf{c}\|=\left\{\begin{array}{l}
\inf _{\alpha \in[0,1]}\left\{\exists m \in \mathcal{M}_{\alpha} \cap \mathcal{M}(\mathbf{c}, f) \text { and } \sigma_{\mathbf{F}}^{*}(\mathbf{c}, m \Phi, T) \leq \alpha\right\} \\
1 \quad \text { otherwise. }
\end{array}\right.
$$

Proposition 8. Let $\Phi=\left\{\phi_{k}\right\}_{k=1}^{\infty}$ be a $T$-(M, F)-representation system of the space $L_{[0,1]}^{0}$. For any $f \in L_{[0,1]}^{0}$ define

$$
\|f\|_{\star}=\inf _{\mathbf{c} \in C_{f}(\Phi)}\|\mathbf{c}\| .
$$

Then $\|\cdot\|_{\star}$ is a quasi-norm on $M / M_{0}$.

Proof. It is clear that

$$
\|f\|_{\star}=0 \Leftrightarrow f \text { is the trivial element of } L_{[0,1]}^{0} .
$$

To check the triangular inequality

$$
\|f+g\|_{\star} \leq\|f\|_{\star}+\|g\|_{\star}
$$

we should consider only the non-trivial case, when $s=\|f\|_{\star}+\|g\|_{\star}<1$. In this case we take arbitrary $\varepsilon>0$ such that $2 \varepsilon<1-s$. We have that there exist $m^{\prime} \in \mathcal{M}_{\|f\|_{\star}+\varepsilon} \cap$ $\mathcal{M}\left(\mathbf{c}^{\prime}, f\right), m^{\prime \prime} \in \mathcal{M}_{\|g\|_{\star}+\varepsilon} \cap \mathcal{M}\left(\mathbf{c}^{\prime \prime}, g\right)$ and such that

$$
\sigma_{\mathbf{F}}^{*}\left(\mathbf{c}^{\prime}, m^{\prime} \Phi, T\right) \leq\|f\|_{\star}+\varepsilon \quad \text { and } \quad \sigma_{\mathbf{F}}^{*}\left(\mathbf{c}^{\prime \prime}, m^{\prime \prime} \Phi, T\right) \leq\|g\|_{\star}+\varepsilon .
$$

Evidently, $m^{\prime} \cdot m^{\prime \prime} \in \mathcal{M}_{s+2 \varepsilon} \cap \mathcal{M}\left(\mathbf{c}^{\prime}, f\right) \cap \mathcal{M}\left(\mathbf{c}^{\prime \prime}, g\right)$ and

$$
\sigma_{\mathbf{F}}^{*}\left(\mathbf{c}^{\prime}+\mathbf{c}^{\prime \prime}, m^{\prime} \cdot m^{\prime \prime} \Phi, T\right) \leq\|f\|_{\star}+\|g\|_{\star}+2 \varepsilon .
$$

Hence,

$$
\|f+g\|_{\star} \leq\|f\|_{\star}+\|g\|_{\star}+2 \varepsilon
$$

and thus the inequality (6) is true. One can easily check that

$$
\|-f\|_{\star}=\|f\|_{\star}, \lim _{\alpha_{n} \rightarrow 0}\left\|\alpha_{n} f\right\|_{\star}=0, \text { and } \lim _{\left\|f_{n}\right\|_{\star} \rightarrow 0}\left\|\alpha f_{n}\right\|_{\star}=0 .
$$


Suppose that $\left\{f_{n}\right\}_{n=1}^{\infty}$ is fundamental with respect to the quasi-norm $\|\cdot\|_{\star}$. Let $\left\{\varepsilon_{k}\right\}_{k=1}^{\infty}$ be a decreasing sequence of positive numbers such that $\forall m \in \mathbf{N}$

$$
\sum_{k=m+1}^{\infty} \varepsilon_{k}<\frac{1}{2} \varepsilon_{m} ; \quad \varepsilon_{1}<1 / 4 .
$$

We can find an increasing sequence of natural numbers $\left\{N_{k}\right\}_{k=1}^{\infty}$ such that

$$
\left\|f_{n}-f_{m}\right\|_{\star}<\varepsilon_{k} \quad \text { for all } n, m \geq N_{k} \text {. }
$$

Hence,

$$
\left\|f_{k}^{*}-f_{k+1}^{*}\right\|_{\star}<\varepsilon_{k} \quad \text { for all } k \in \mathbf{N}
$$

if we define

$$
f_{k}^{*}=f_{N_{k}}, \quad \text { for all } k \in \mathbf{N} .
$$

This means that for any $k \in \mathbf{N}$ there exist $\mathbf{c}^{(k)} \in C_{f_{k}^{*}-f_{k+1}^{*}}(\Phi)$ and $m_{k} \in \mathcal{M}_{\varepsilon_{k}} \cap$ $\mathcal{M}\left(\mathbf{c}^{(k)}, f_{k}^{*}-f_{k+1}^{*}\right)$ such that

$$
\lim _{n \rightarrow \infty} \sigma_{n}\left(\mathbf{c}^{(k)}, m_{k} \Phi, T\right)(\cdot) \stackrel{\mathbf{F}}{=} m_{k}(\cdot)\left(f_{k}^{*}(\cdot)-f_{k+1}^{*}(\cdot)\right), \quad \sigma_{\mathbf{F}}^{*}\left(\mathbf{c}^{(k)}, m_{k} \Phi, T\right) \leq \varepsilon_{k} .
$$

Denote

$$
m^{(k)}(x)=\prod_{j=k}^{\infty} m_{j}(x) .
$$

Evidently, $m^{(k)} \in \mathcal{M}_{\varepsilon_{k-1}}$. It is obvious that for any $\ell>k$

$$
\lim _{n \rightarrow \infty} \sigma_{n}\left(\sum_{i=k}^{\ell} \mathbf{c}^{(i)}, m^{(k)} \Phi, T\right)(\cdot) \stackrel{\mathbf{F}}{=} m^{(k)}(\cdot)\left(f_{k}^{*}(\cdot)-f_{\ell}^{*}(\cdot)\right),
$$

and

$$
\sigma_{\mathbf{F}}^{*}\left(\sum_{i=k}^{\ell} \mathbf{c}^{(i)}, m^{k} \Phi, T\right) \leq \varepsilon_{k-1}
$$

For any $n \in \mathbf{N}$ by $q_{n}$ we denote the minimal natural number such that

$$
\alpha_{i}=\sum_{j=i}^{q_{n}} t_{q_{n} j} \neq 0 \quad \text { for all } \quad i \in[1, n] \cap \mathbf{N} .
$$

Evidently $q_{1}=1$ and $\left\{q_{n}\right\}_{n=1}^{\infty}$ is a non-decreasing sequence. Next we examine the sets of functions

$$
\phi_{q_{n}+1}, \phi_{q_{n}+2}, \ldots, \phi_{q_{n+1}}, \quad \text { for all } n \in \mathbf{N},
$$

and deleting, if necessary, the functions with greatest indices, we obtain linearly independent collections of functions such that any element from (8) is a finite linear combination of remaining functions. For simplicity, we will conserve the notation and consider that for any $n \in \mathbf{N}$ the functions (8) are linearly independent.

If we fix any $\nu \in \mathbf{N}$ then for any $\ell, k \in \mathbf{N}$ and $\ell>k>\nu$

$$
\lim _{n \rightarrow \infty} \sigma_{n}\left(\sum_{i=k}^{\ell} \mathbf{c}^{(i)}, m^{(\nu)} \Phi, T\right)(\cdot) \stackrel{\mathbf{F}}{=} m^{(\nu)}(\cdot)\left(f_{k}^{*}(\cdot)-f_{\ell+1}^{*}(\cdot)\right),
$$


and

$$
\sigma_{\mathbf{F}}^{*}\left(\sum_{i=k}^{\ell} \mathbf{c}^{(i)}, m^{\nu} \Phi, T\right) \leq \varepsilon_{k-1} .
$$

From the above conditions and the linear independence of functions (8) it is easy to check that there exists $g_{\nu} \in \mathbf{F}$ such that

$$
\lim _{n \rightarrow \infty} \sigma_{n}\left(\mathbf{b}^{(\nu)}, m^{(\nu)} \Phi, T\right)(\cdot) \stackrel{\mathbf{F}}{=} m^{(\nu)}(\cdot) f_{k}^{*}(\cdot)-g_{\nu}(\cdot)
$$

and

$$
\sigma_{\mathbf{F}}^{*}\left(\mathbf{b}^{(\nu)}, m^{\nu} \Phi, T\right) \leq \varepsilon_{k-1},
$$

where

$$
\mathbf{b}^{(\nu)}=\left(b_{j}^{(\nu)}\right)_{j=1}^{\infty} \quad \text { and } \quad b_{j}^{(\nu)}=\sum_{i=\nu}^{\infty} c_{j}^{(i)} .
$$

From (7) we have that the sets $E_{k}=\left\{x \in[0,1]: m^{(k)}(x)=1\right\}$ constitute an increasing sequence of sets that tend to a set of complete measure, hence, if we define

$$
f(x)=g_{\nu}(x) \quad \text { if } \quad x \in E_{\nu} \backslash \bigcup_{n=0}^{\nu-1} E_{n} \quad \text { for any } \nu \in \mathbf{N}
$$

where $E_{0}=\emptyset$, then $f$ will be a measurable and a.e. finite function on $[0,1]$. From (9) and (10) follows that

$$
\lim _{k \rightarrow \infty}\left\|f_{k}^{*}-f\right\|_{\star}=0
$$

Thus $\|\cdot\|_{\star}$ is a quasi-norm on $M / M_{0}$.

TheOREM 9. Let $\Phi=\left\{\phi_{k}\right\}_{k=1}^{\infty}$ be a $T-(M, \mathbf{F})$-representation system of the space $L_{[0,1]}^{0}$. Then for any $N \in \mathbf{N}$ the system $\Phi_{N}=\left\{\phi_{k}\right\}_{k=N+1}^{\infty}$ is also a $T-(M, \mathbf{F})$-representation system of the space $L_{[0,1]}^{0}$.

Proof. The linear space of Lebesgue measurable functions defined on the interval $[0,1]$ with the quasi-norm $\|\cdot\|_{\star}$ is an $F$-space. Denote that space by $L_{[0,1]}^{*}$. Evidently, the theorem will be proved if we prove it for the case $N=1$. Suppose that the system $\Phi_{1}=\left\{\phi_{k}\right\}_{k=2}^{\infty}$ is not a $T$ - $(M, \mathbf{F})$-representation system of the space $L_{[0,1]}^{*}$. It means that for any $f \in L_{[0,1]}^{*}$ only one of the following cases is possible: $\operatorname{card} C_{f}(\Phi)=1$ or $\operatorname{card} C_{f}(\Phi)>1$ and for any $\mathbf{c}, \mathbf{c}^{\prime} \in C_{f}(\Phi)$ we have that $c_{1}=c_{1}^{\prime}$, where $\mathbf{c}=\left(c_{1}, c_{2}, \ldots\right)$ and $\mathbf{c}^{\prime}=\left(c_{1}^{\prime}, c_{2}^{\prime}, \ldots\right)$. Hence, for any $f \in L_{[0,1]}^{*}$ and any $\mathbf{c} \in C_{f}(\Phi), c_{1}(f)$ is defined uniquely and can be considered as a linear functional on $L_{[0,1]}^{*}$. By (5) it can be easily checked that $c_{1}(f)$ will be also continuous on $L_{[0,1]}^{*}$. Then, by a well known standard method, applying Lemma 7 one shows that $c_{1}(f)$ will be a non-trivial linear continuous functional on the space $L_{[0,1]}^{0}$. This contradicts the well known fact that the dual of $L_{[0,1]}^{0}$ consists uniquely of the trivial element.

The following lemma permits to obtain Proposition 4 as a consequence of Theorem 9. LEMMA 10. Let $\left\{g_{k}\right\}_{k=1}^{\infty} \subset M$ be a sequence which converges a.e. to $g \in M$. Then there exists $m \in L_{[0,1]}^{\infty}, 0<m(x) \leq 1$ such that

$$
\lim _{k \rightarrow \infty}\left\|m\left(g_{k}-g\right)\right\|_{\infty}=0 .
$$


Proof. Let $\varepsilon_{n} \downarrow 0$ be a decreasing sequence of positive numbers such that $\sum_{n=1}^{\infty} \varepsilon_{n}<1$. By Egorov's theorem for any $\varepsilon_{n}, n \in \mathbf{N}$ we can find $N_{n} \in \mathbf{N}$ and a bounded measurable function $m_{n}(x), 0<m_{n}(x) \leq 1$ such that

$$
\left|\left\{x \in[0,1]: m_{n}(x)=1\right\}\right|>1-\varepsilon_{n},
$$

and

$$
\left\|m_{n}\left(g_{k}-g\right)\right\|_{\infty}<\varepsilon_{n} \quad \text { for all } \quad k>N_{n} .
$$

Then we define $m(x)=\prod_{n=1}^{\infty} m_{n}(x)$ and easily check that (11) holds.

Proposition 3 is an immediate corollary of Proposition 4.

\section{References}

[1] S. Banach, Theory of Linear Operations, North-Holland, Amsterdam, 1987.

[2] C. Bennett and R. Sharpley, Interpolation of Operators, Academic Press, London, 1988.

[3] S. Kaczmarz and H. Steinhaus, Theorie der Orthogonalreihen, Monografie Mat., Warszawa-Lwów, 1935.

[4] B. S. Kashin, A certain complete orthonormal system, Mat. Sb. 99 (141) (1976), no. 3, 356-365 (in Russian); English transl.: Math. USSR Sb. 28 (1976), 315-324.

[5] K. S. Kazarian and D. Waterman, Theorems on representations of functions by series, Mat. Sb. 191 (2000), no. 12, 123-140 (in Russian); English transl.: Sb. Math. 191 (2000), no. 11-12, 1873-1889.

[6] K. Kazarian, A complete orthonormal system of divergence, J. Functional Analysis 214 (2004), 284-311.

[7] K. Kazarian, The zero-one law for a complete orthonormal system, C.R. Acad. Sci. Paris 339, (2004), 335-337.

[8] K. Kazarian, A simple complete orthonormal system of functions, to appear.

[9] N. N. Lusin, Integral and trigonometric series, editing and commentary by N. K. Bari and D. E. Menshov. Gos. Izdat. Tehn.-Teor. Lit., Moscow-Leningrad, 1951 (in Russian).

[10] D. Menchoff (D. E. Menshov), Sur les représentations des fonctions mesurables par des séries trigonométriques, Rec. Math. [Mat. Sbornik] N. S. 9 (51) (1941), 667-692.

[11] A. A. Talalian, Problems on representation and unicity in the theory of orthogonal series, Itogi nauki, Matematicheskii Analiz, VINITI, 1971, Moscow, 3-64 (in Russian).

[12] K. Yosida, Functional Analysis, Springer-Verlag, Berlin, 1980.

[13] A. Zygmund, Trigonometric Series, Vol. 1, Cambridge Univ. Press, 1959. 